\title{
Microwave dependence of subharmonic gap structure in superconducting junctions
}

\author{
Sørensen, O. Hoffman; Kofoed, Bent; Pedersen, Niels Falsig; Shapiro, Sidney
}

Published in:

Physical Review B

Link to article, DOI:

10.1103/PhysRevB.9.3746

Publication date:

1974

Document Version

Publisher's PDF, also known as Version of record

Link back to DTU Orbit

Citation (APA):

Sørensen, O. H., Kofoed, B., Pedersen, N. F., \& Shapiro, S. (1974). Microwave dependence of subharmonic gap structure in superconducting junctions. Physical Review B, 9(9), 3746-3756.

https://doi.org/10.1103/PhysRevB.9.3746

\section{General rights}

Copyright and moral rights for the publications made accessible in the public portal are retained by the authors and/or other copyright owners and it is a condition of accessing publications that users recognise and abide by the legal requirements associated with these rights.

- Users may download and print one copy of any publication from the public portal for the purpose of private study or research.

- You may not further distribute the material or use it for any profit-making activity or commercial gain

- You may freely distribute the URL identifying the publication in the public portal 


\title{
Microwave dependence of subharmonic gap structure in superconducting junctions
}

\author{
O. Hoffmann Soerensen, B. Kofoed, N. F. Pedersen, and Sidney Shapiro* \\ Physics Laboratory I, The Technical University of Denmark, DK-2800, Lyngby, Denmark
}

(Received 23 August 1973)

\begin{abstract}
Experiments on both point-contact junctions $(\mathrm{Nb}-\mathrm{Nb})$ and on small area thin-film tunnel junctions (Sn-O-Sn) show that applied 4-mm radiation produces satellites associated with "subharmonic" gap structure as well as the familiar microwave-assisted tunneling structure associated with the superconducting energy gap itself. The location in voltage of all these structures is given by $e V=(2 \Delta \pm n h v) / m$, where $2 \Delta$ is the superconducting energy gap, $v$ is the applied frequency, $h$ is Planck's constant, $e$ is the magnitude of the electronic charge, $V$ is the dc voltage drop across the junction, and $m$ and $n$ are integers: $m=1,2,3, \ldots$ and $n=0,1,2, \ldots$. The power dependence of the satellite structure and the microwave-assisted tunneling structure is consistent for all junctions tested with the expression $J_{n}^{2}\left(m e V_{\mathrm{rf}} / h v\right)$, where $J_{n}(x)$ is the ordinary Bessel function of order $n, V_{\mathrm{rf}}$ is the amplitude of the induced microwave voltage drop accross the junction, and the other quantities are as defined above. A distinction between the explanations-multiparticle tunneling and nonlinear self-coupling - of the subharmonic gap structure cannot, however, be made on the basis of the observed power dependence. Hence other properties such as relative amplitude and line shape are considered and it is concluded that self-coupling mechanisms seem able to account for all the observations, whereas multiparticle tunneling may be discarded as the sole explanation of the structure.
\end{abstract}

\section{INTRODUCTION}

In the past decade considerable effort has been put into attempts to understand the so-called "subharmonic" gap structure in the current-voltage (IV) characteristic of superconducting junctions. ${ }^{1-20}$ It is well known that structure is commonly observed at voltages approximately equal to submultiples of the superconducting energy gap, that is, at voltages $2 \Delta / m e$, where $2 \Delta$ is the energy gap, $\boldsymbol{e}$ is the magnitude of the electronic charge, and $m=1,2,3, \ldots$. This structure is seen in thin-film tunnel junctions ${ }^{1-12}$ as well as in point-contact junctions, ${ }^{13-16}$ but the origin of the structure is not yet fully understood. Various explanations have been suggested such as multiparticle tunneling, ${ }^{17}$ nonlinear self-coupling of the Josephson radiation, ${ }^{18}$ breaking of $p$ pairs and the simultaneous tunneling of $n$ electrons $(p<n),{ }^{19}$ and pair breaking in the barrier. ${ }^{20}$

In order to obtain further experimental insight into the phenomenon we have made a careful series of measurements of the microwave-power ( $\mathrm{rf}$-power) dependence of the subharmonic gap structure (SGS) for point-contact junctions as well as for thin-film tunnel junctions. The experiments show that the amplitude of the SGS at $V=2 \Delta / m e$ varies with $\mathrm{rf}$ power as $J_{0}^{2}(m \alpha)$; here $J_{0}(x)$ is the ordinary Bessel function of order zero, and $\alpha=e V_{\mathrm{r} f} / h \nu$, where $V_{\mathrm{r} f}$ is the induced microwave voltage drop across the junction, $h$ is Planck's constant, and $\nu$ is the applied frequency. In addition, satellite structures are observed at voltages $(2 \Delta \pm n h \nu) / m e$, where $n=1,2,3, \ldots$ These satellites vary with $\mathrm{rf}$ power as $J_{n}^{2}(m \alpha)$, where $J_{n}(x)$ is the ordinary Bessel function of order $n$. All the junctions tested be- haved consistently with these results. ${ }^{21}$

In the companion paper by Hasselberg, Levinsen, and Samuelsen ${ }^{22}$ some suggested explanations of the SGS are analyzed, and it is shown that both multiparticle tunneling and nonlinear self-coupling yield an $\mathrm{rf}$-power dependence in agreement with the experimental results. The present paper is organized along the following lines: In Sec. II the experimental details are presented. In Sec. III A the most complete experimental results on power dependence are presented both for point-contact junctions (subsection 1) and for thin-film tunnel junctions (subsection 2). In Sec. III B a data-reduction procedure is described, and in Sec. III C the reduced data are compared to theory. The rf results are discussed in Sec. III D. In Sec. IV other experimental results are briefly presented and discussed, and Sec. V consists of a summary and the conclusion.

\section{EXPERIMENTAL DETAILS}

\section{A. Experimental setup}

The samples were niobium-niobium $(\mathrm{Nb}-\mathrm{Nb})$ point contacts and tin-tin-oxide-tin ( $\mathrm{Sn}-O-\mathrm{Sn})$ thinfilm tunnel junctions. The thin-film tunnel junctions were small area cross-type junctions. Full details of their preparation will be given elsewhere. ${ }^{23}$ Here we note only that the Sn films were deposited on glass slides by evaporation in vacuum $\left(\sim 10^{-6}\right.$ torr) and that the barrier was produced by letting the first deposited film oxidize for about $1 \frac{1}{2} \mathrm{~h}$ in an atmosphere of pure dry oxygen at $50^{\circ} \mathrm{C}$. The effective tunneling area was about $1 \times 20 \mu \mathrm{m}$ thus producing low-capacitance junctions with $I V$ characteristics typically as shown in Fig. 1. The point contacts were made between a $0.5-\mathrm{mm}$-diam 


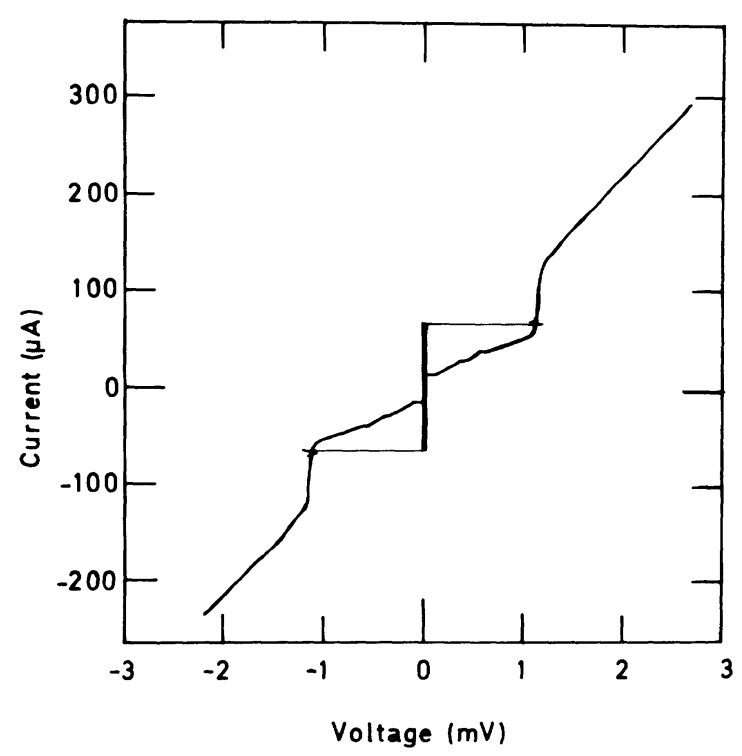

FIG. 1. Current-voltage characteristic for $\mathrm{Sn}-\mathrm{O}-\mathrm{Sn}$ thin-film tunnel junction at $T=1.6 \mathrm{~K}$. Reproduced photographically from oscilloscope display. The load-line switching is due to the junction being current biased. The rather small hysteresis is due to the low capacitance of this small-area junction $(1 \times 20 \mu \mathrm{m})$. Normal-state resistance $9.2 \Omega$.

pointed $\mathrm{Nb}$ wire and a $2.5 \times 3.0 \times 0.2-\mathrm{mm} \mathrm{Nb}$ flat. The wire was sharpened electrolytically ${ }^{24}$ in a $5: 4: 1$ solution of $\mathrm{HNO}_{3}, \mathrm{HF}$, and $\mathrm{CH}_{3} \mathrm{COOH}$ producing points with a radius of curvature as small as $1 \mu \mathrm{m}$ as observed by inspection in a microscope. However, usually points with a radius of curvature of order $10 \mu \mathrm{m}$ were used. The flat was polished with No. 400 emery cloth and etched slightly in the solution mentioned above. By exclusively using samples with small cross-sectional areas, the complications associated with spatial variation of the induced microwave fields are avoided. ${ }^{25,26}$

The microwave system consisted of a $4-\mathrm{mm}$ klystron (OKI70V10), a ferrite isolator, a directional coupler mounted with a crystal detector for monitoring the power output, a buffer attenuator, and a calibrated precision attenuator. The measurements were done in a shielded room, and in order to further minimize noise the klystron and the klystron power supply were mounted outside this room.

The double-glass-Dewar system was surrounded by a two-layered $\mathrm{Mu}$-metal can to screen out the earth's magnetic field, and a pair of Helmholtz coils was placed in the liquid-nitrogen Dewar providing magnetic fields up to about $50 \mathrm{Oe}$, if desired.

For the thin-film tunnel-junction measurements, an experimental setup was used in which the tunnel junction was mounted just below an open-ended waveguide and directly immersed into the helium bath. The vertical distance between the junction and the waveguide and the angle between the junction plane and the waveguide axis could both be adjusted from outside the cryostat to optimize the coupling to the microwave fields.

A different experimental rig, to be described in detail elsewhere, ${ }^{27}$ was used for the point-contact measurements. Here the pointed $\mathrm{Nb}$ wire was led through the broad wall of the $4 \mathrm{~mm}$ waveguide, and was electrically insulated from it by a carbonimpregnated high-frequency -absorber material (Eccosorb) in order to quench the $Q$ of the coaxial cavity formed by the waveguide wall and the $\mathrm{Nb}$ wire. The flat was mounted on a phosphor-bronze "leaf spring" which formed the waveguide wall opposite the point. The vertical motion of a 15:1 differential screw was converted to a horizontal displacement of the $\mathrm{Nb}$ flat via a $15: 1$ excentric lever mechanism pressing against the leaf spring. The differential screw was situated immediately above the point contact and was immersed directly into liquid helium in order to avoid instabilities due to thermal strains. This arrangement had fine adjustment capabilities and produced very stable contacts. A given setting of the point contact was once maintained for $7 \mathrm{~h}$ without any observable change in the $I V$ characteristic or in its derivative until the contact was deliberately readjusted. However, long-term stability of this order is not needed in order to perform experiments of the type to be reported here. In order to complete successfully an experimental run the point-contact setting should be maintained only for about 1 to $2 \mathrm{~h}$ and this requirement was met quite readily. Three representative $I V$ characteristics corresponding to different point-contact adjustments are shown in Fig. 2.

\section{B. Experimental procedure}

As is evident from Figs. 1 and 2, the SGS is not sufficiently pronounced in the $I V$ characteristics that quantitative results can be obtained directly from them. However, by using standard ac-modulation techniques for recording of derivatives, the SGS was well resolved (see, e.g., Fig. 3). Corresponding to whether approximately constantvoltage or constant-current bias was used, the differential conductance $d I / d V$ or the differential resistance $d V / d I$ was recorded. In every case the ac-modulation amplitude was chosen small enough that the observed line shape was independent of the modulation. Although the differential conductance is more readily related to theory, the differential resistance was favorable with respect to resolution of the satellite structures. The reason for this, which relates to the problems connected 


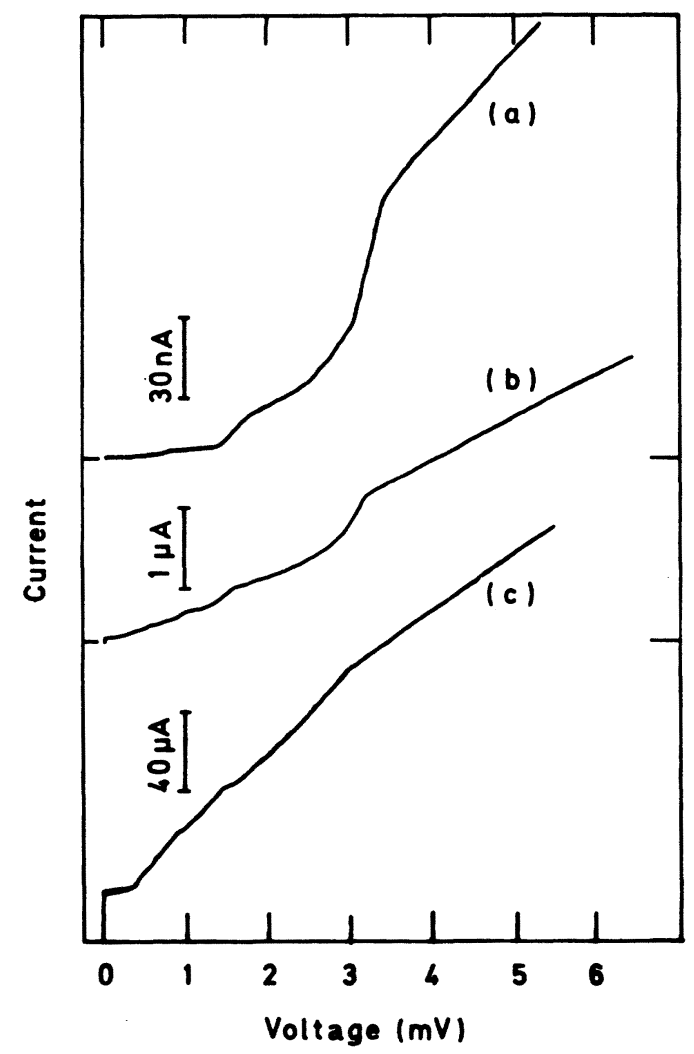

FIG. 2. Current-voltage characteristics for $\mathrm{Nb}-\mathrm{Nb}$ point-contact junctions reproduced from $X Y$-recorder traces. The traces all start from zero current and the current scales are as indicated by the bars. (a) Normalstate resistance $33 k \Omega$, no observable zero-voltage current; (b) normal-state resistance $1.9 \mathrm{k} \Omega$, zero-voltage current $50 \mathrm{nA}$; (c) normal-state resistance $29 \Omega$, zerovoltage current $25 \mu \mathrm{A}$. Temperature $T=4.2 \mathrm{~K}$.

with the indexing of the structures, is discussed briefly in Sec. IV.

During experimental runs the derivative, either $d I / d V$ or $d V / d I$, was recorded on an $X Y$ recorder vs bias voltage as a function of incoming $\mathrm{rf}$ power. The calibrated attenuator reading was the measure of the power level, and the power was in turn assumed proportional to the induced-rf-voltage squared, $V_{\mathrm{rf}}^{2}$. Thus, the obtained power dependence could-by adjustment of the scaling factor between rf power and $V_{\text {rf }}^{2}$-be compared directly with theory. Since the theory yields an expression for the current or the conductance as a function of bias and $\mathrm{rf}$-voltage (see Sec. III B), structure in resistance traces was transformed to structure in conductance before comparison was made. Finally, it is stressed that in order to assure that reliable data were obtained, the setting of the point contact was checked several times during each complete run by retracing of the zero-rf-power derivative.

\section{MICROWAVE EXPERIMENTS}

\section{A. Experimental results \\ 1. Point-contact junctions}

Figure 3 shows, as a function of bias voltage, typical examples of derivative $X Y$-recorder traces for $\mathrm{Nb}-\mathrm{Nb}$ point contacts with no applied $\mathrm{rf}$. Figure 3(a) shows differential conductance $d I / d V$ for one point contact with normal-state resistance $54 \Omega$, and Fig. 3(b) shows differential resistance $d V / d I$ for another point contact with normal-state resistance $27 \Omega$. In the conductance trace the gap structure and the SGS show up as peaks which in the case of the half-gap and the one-third-gap structures are broad compared to the spacing of the $\mathrm{rf}$-induced satellites as indicated by the bars in the figure. In the resistance trace the gap structure appears as a sharp steplike rise in resistance, whereas the subharmonics have developed

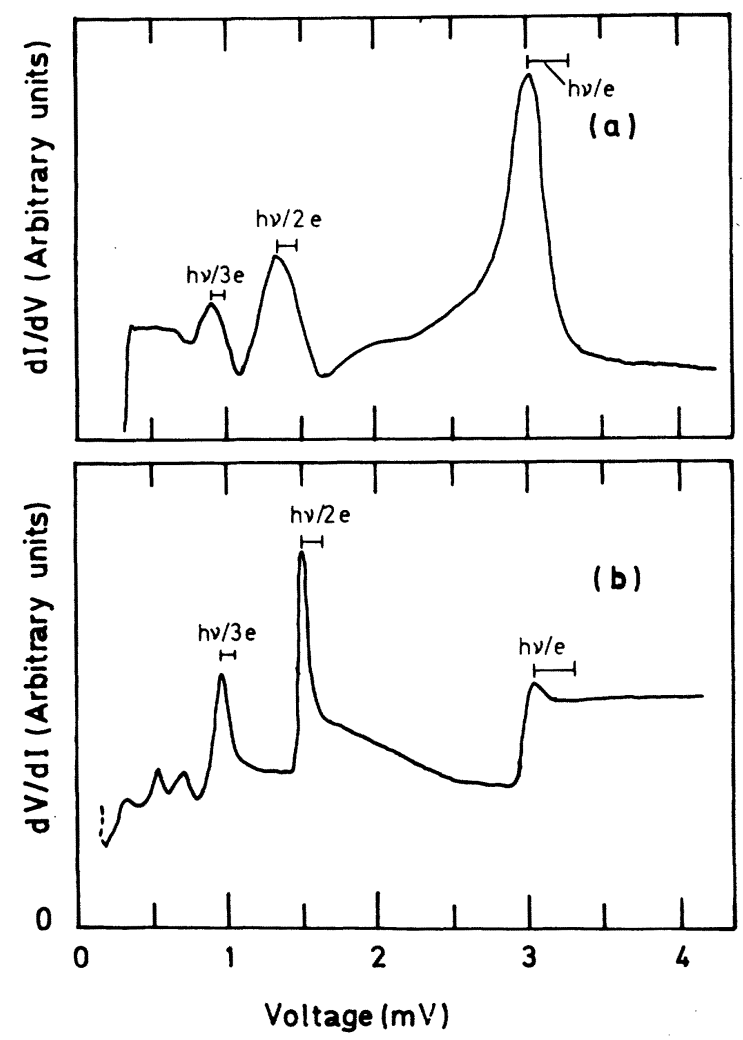

FIG. 3. (a) Differential conductance of $\mathrm{Nb}-\mathrm{Nb}$ pointcontact junction showing subharmonic gap structure. The bars at the peaks show the satellite separation corresponding to applied $4-\mathrm{mm}$ radiation $(66 \mathrm{GHz})$. The zero conductance level has been suppressed. (b) Differential resistance of the $\mathrm{Nb}-\mathrm{Nb}$ point-contact junction of Fig. 2 (c). The subharmonic gap structure is clearly resolved. The bars at the peaks indicate the satellite separation as in $3(\mathrm{a})$. The base line corresponds to zero differential resistance. Temperature $T=4.2 \mathrm{~K}$. 
into narrow resistance peaks, which-especially for the half-gap peak-are sharp compared to the satellite separation. In fact, it was observed as a rule that the SGS always appeared sharper in resistance compared to conductance recordings.

Figure 4 shows an almost complete sequence of recordings of conductance vs bias voltage as a function of rf power (a few traces have been omitted for the sake of clarity). Each trace corresponds to a different level of $\mathrm{rf}$ power as given by the calibrated-attenuator reading, and the uppermost trace (at $-34 \mathrm{~dB}$ ) is indistinguishable from the zero-power trace shown as Fig. 3(a) for the same point-contact setting. Consider the variation of the conductance peak at the gap voltage $(m=1)$ as the power is increased: it is seen first to decrease, apparently to zero at some power level, then to increase, and once again to decrease. The halfgap peak $(m=2)$, however, decreases at a faster rate and the one-third-gap peak $(m=3)$ decreases even faster with increasing power. Also observed is the well-known microwave-assisted-tunneling (MAT) effect ${ }^{28}$ on either side of the gap structure. Here the rf-induced replicas of the gap peak corresponding to $n=1,2,3$, and 4 are clearly resolved, and their spacing, $h \nu / e=270 \mu \mathrm{V}$, is in agreement with theory. ${ }^{18,29}$ The satellite structures to the subharmonic gap structures are not clearly resolved because their widths, as noted above, are comparable to the satellite separation, $h \nu / m e$. However, as seen in Fig. 4, when the half-gap peak disappears two peaks remain on either side of the van-

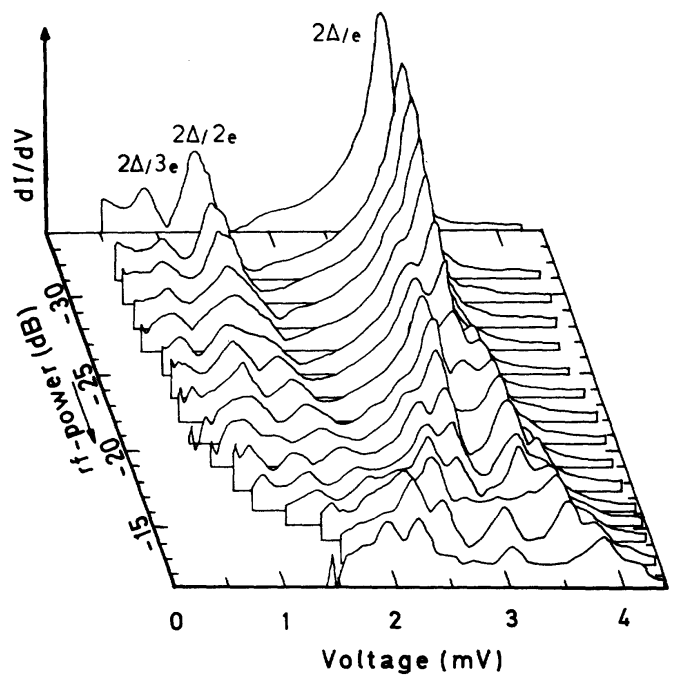

FIG. 4. Sequence of differential-conductance traces as a function of $\mathrm{rf}$ power and bias voltage for the $\mathrm{Nb}-\mathrm{Nb}$ point contact of Fig. 3(a). The zero-conductance level has been suppressed and the low-voltage parts showing rf-induced Josephson steps have been omitted for the sake of clarity.

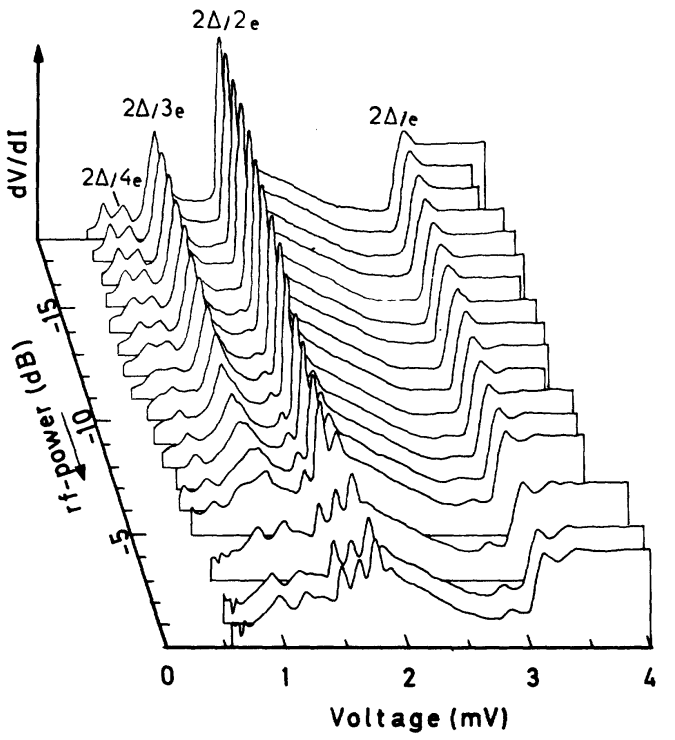

FIG. 5. Sequence of differential resistance traces as a function of $\mathrm{rf}$ power and bias voltage for the $\mathrm{Nb}-\mathrm{Nb}$ point contact of Fig. $3(\mathrm{~b})$. As in Fig. 4 the low-voltage parts of the traces have been omitted. The zero-resistance level is not included in the figure but can be determined by comparison with Fig. 3(b).

ishing peak, and their separation is twice $h \nu / 2 e$ as the distance between the two $n=1$ peaks should be.

Figure 5 shows the complete sequence of resis tance recordings as a function of bias voltage and rf power for the point contact of Fig. 2(c) [and of Fig. 3(b)]. Again the MAT effect is observed at the gap voltage. Although only moderate $\mathrm{rf}$ powers were attained in this case, the resistance rise does start to decrease and replicas start to show up on both sides. But more important, due to the sharpness of the SGS, the satellites $(n=1)$ at the halfgap structure are resolved and also the one-thirdgap structure is seen to split into two peaks spaced by $2(h \nu / 3 e)$ as the power increases. Finally, note that the rate of decrease of the SGS is higher the larger the $m$ value, as was the case also for the sequence of Fig. 4.

\section{Thin-film tunnel junctions}

The IV characteristic of Fig. 1 is shown also in Fig. 6 on an expanded current scale. Here the $m=2$ and 3 subharmonic gap structures show up as very sharp cusps in the current confined to a narrow voltage range. The sweep is current controlled and hence the SGS is only traced out when the current is swept towards zero from above due to the load-line switching. Unfortunately this switching back to the zero-voltage state-which in Fig. 6 occurs at a bias voltage about $0.1 \mathrm{mV}-$ occurred just below the $m=2$ structure when the 


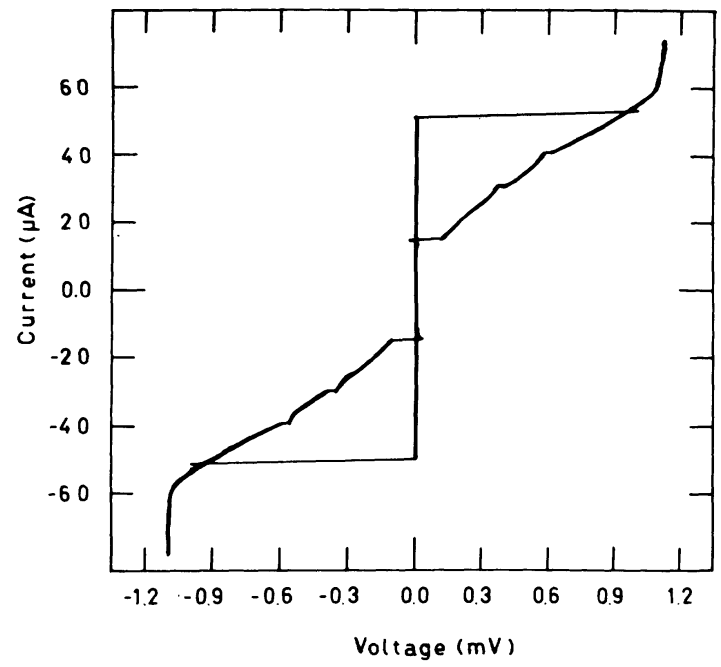

FIG. 6. Current-voltage characteristic of Fig. 1 shown on expanded scales. The subharmonic gap structures $m=2$ and $m=3$ are very narrow current cusps of nearly equal magnitude.

derivative was traced out on the $X Y$ recorder. Hence, only the $m=1$ and 2 structures are shown in Fig. 7, where a selection of the resistance traces is presented as a function of bias voltage and $\mathrm{rf}$ power. The gap structure appears as a resistance dip and the half-gap structure as a resistance dip followed by a very sharp spike reflecting the high differential resistance on the high-voltage side of the current cusp. As the rf power increases, the $n=1$ structures appear as replicas of the original $n=0$ structure, and most important to note is that the structures are all well separated so that overlap effects are negligibly small.

Figure 8 shows a selection of conductance recordings for another Sn-O-Sn thin-film tunnel junction. Here the SGS appears as conductance peaks and again all structures are well separated. In this case also the $m=3$ peak is included on the lowpower traces, but its variation with $\mathrm{rf}$ power can only be followed over a moderate power range because of switching to the zero-voltage state.

\section{B. Data analysis}

The data presented so far clearly exhibit ordinary MAT. The Tien-Gordon expression ${ }^{29}$ for MAT in small junctions where spatial variation of the $\mathrm{rf}$ fields can be neglected,

$$
I_{r f}(V)=\sum_{n=-\infty}^{\infty} J_{n}^{2}(\alpha) I_{0}(V+n h \nu / e)
$$

has been verified in great detail. ${ }^{25,26,30} I_{r f}(V)$ is the current through the contact in the presence of rf and $I_{0}(V)$ is the current with no applied rf. Equa- tion (1) can readily be extended to include also the dependence of the SGS on microwave radiation. The following qualitative results have been obtained from Figs. 4-8: (i) The SGS is increasingly sensitive to $\mathrm{rf}$ power as the index number $m$ increases and (ii) the $\mathrm{rf}$-induced satellite structures are evidently spaced in voltage by $h \nu / m e$. Accordingly, a natural generalization of Eq. (1) is

$$
I_{\mathrm{rf}}(V)=\sum_{m=1}^{\infty} \sum_{n=-\infty}^{\infty} J_{n}^{2}(m \alpha) I_{m}(V+n h \nu / m e) .
$$

$I_{m}(V)$ is the current contribution responsible for the $m$ th SGS with no applied $\mathrm{rf}$, and the remaining quantities are as defined above. The term $m=1$ is recognized as the Tien-Gordon expression. We postulate ${ }^{31}$ that Eq. (2) is valid also for $m \geqq 2$. Dif ferentiation of Eq. (2) with respect to $V$ yields the differential conductance $\sigma(V)$ which satisfies

$$
\sigma_{\mathrm{rf}}(V)=\sum_{m} \sum_{n} J_{n}^{2}(m \alpha) \sigma_{m}(V+n h \nu / m e)
$$

To proceed further this expression is cast into a form more suitable for the data analysis. The SGS is characterized by the localized and readily measurable changes in conductance, $\Delta \sigma_{\mathrm{rf}}$. The conductance change may be defined as

$$
\Delta \sigma_{r f}=\sigma_{r f}\left(V_{1 n}\right)-\sigma_{r f}\left(V_{2 n}\right)
$$

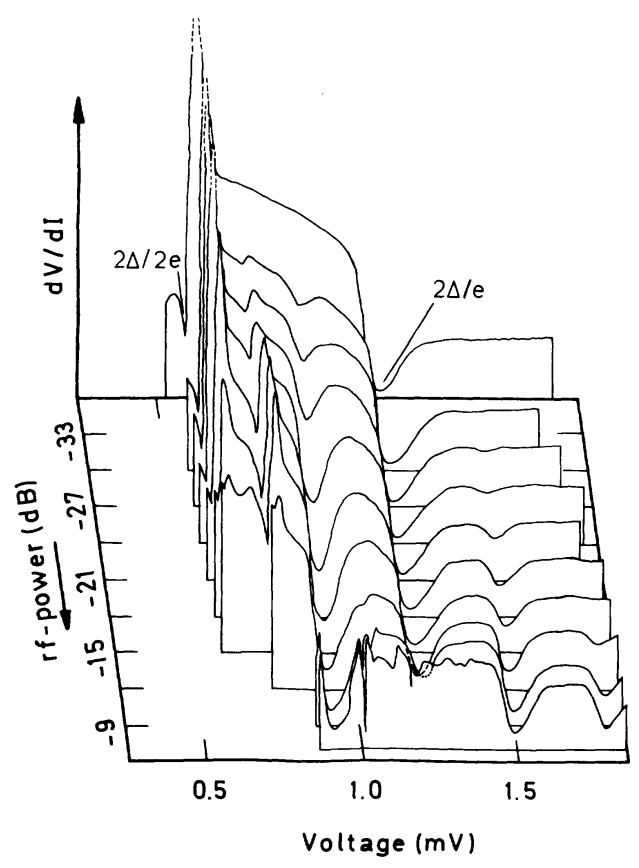

FIG. 7. Sequence of differential resistance traces as a function of $\mathrm{rf}$ power and bias voltage for the $\mathrm{Sn}-\mathrm{O}-\mathrm{Sn}$ tunnel junction of Figs. 1 and 6 . The base plane corresponds to zero resistance. Note that $V=0 \mathrm{mV}$ is not included in the figure. 


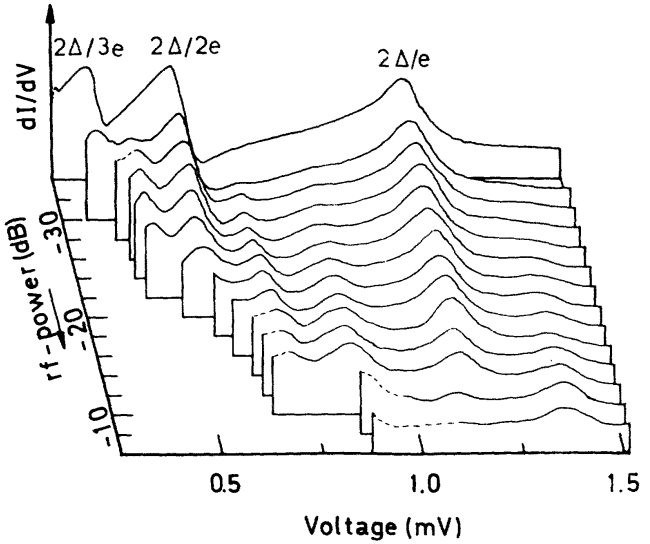

FIG. 8. Sequence of differential conductance traces as a function of $\mathrm{rf}$ power and bias voltage for a $\mathrm{Sn}-\mathrm{O}-\mathrm{Sn}$ tunnel junction at $T=1.6 \mathrm{~K}$. The base plane is arbitrarily chosen, and, as in Fig. $7, V=0 \mathrm{mV}$ is not included.

if a characteristic well-defined feature of the $n$th satellite belonging to the $m$ th SGS is confined within the (narrow) voltage interval $\left(V_{1 n}, V_{2 n}\right)$. Alternatively, $\Delta \sigma_{\mathrm{rf}}$ may be defined as

$$
\Delta \sigma_{\mathrm{rf}}=\sigma_{\mathrm{rf}}\left(V_{1 n}\right)-\sigma_{B},
$$

if the characteristic structure element is a conductance peak which rises above a background level, $\sigma_{B}$. Here, $V_{1 n}$ is the voltage at which the $n$th conductance peak reaches its maximum. $\sigma_{B}$ is, in principle, arbitrary but, as is demonstrated below, the background level is, in most cases, well defined.

The aim of this discussion is to show that the rf dependence of the measurable quantity $\Delta \sigma_{\mathrm{rf}}-\mathrm{de}-$ fined by either of Eqs. (4) or (5) combined with Eq. (3)-is given by $J_{n}^{2}(m \alpha)$, as was first suggested by Longacre and Shapiro. ${ }^{15}$ The method of analysis is then tested on MAT in order to see if the known results are reproduced and, finally, proving satisfactory in this case, the method is applied to the SGS and $\mathrm{rf}$-induced satellites.

If the definition (4) is used, $\Delta \sigma_{\mathrm{rf}}$ is found to be

$$
\begin{aligned}
\Delta \sigma_{\mathrm{rf}}= & \sum_{m} \sum_{n} J_{n}^{2}(m \alpha)\left[\sigma_{m}\left(V_{1 n}+n h \nu / m e\right)\right. \\
& \left.-\sigma_{m}\left(V_{2 n}+n h \nu / m e\right)\right] .
\end{aligned}
$$

Here, all but one term in the $m$ summation cancel if $\sigma_{m}$ is a slowly varying function of $V$ everywhere except when $V$ is close to $2 \Delta / m e$. Hence

$$
\begin{aligned}
\Delta \sigma_{\mathrm{rf}}= & \sum_{n} J_{n}^{2}(m \alpha)\left[\sigma_{m}\left(V_{1 n}+n h \nu / m e\right)\right. \\
& \left.-\sigma_{m}\left(V_{2 n}+n h \nu / m e\right)\right] .
\end{aligned}
$$

Normalizing to the amplitude $\Delta \sigma_{m}=\sigma_{m}\left(V_{10}\right)-\sigma_{m}\left(V_{20}\right)$ of the chosen structure element ${ }^{32}$ when no $\mathrm{rf}$ is applied, the final result is

$$
\begin{aligned}
\frac{\Delta \sigma_{r f}}{\Delta \sigma_{m}}= & \sum_{n} J_{n}^{2}(m \alpha) \\
& \times \frac{\sigma_{m}\left(V_{1 n}+n h \nu / m e\right)-\sigma_{m}\left(V_{2 n}+n h \nu / m e\right)}{\Delta \sigma_{m}} .
\end{aligned}
$$

Provided that terms in Eq. (8) corresponding to different $n$ 's do not overlap in voltage then the SGS $(n=0)$ varies as $J_{0}^{2}(m \alpha)$, and the $n$th satellites vary as $J_{n}^{2}(m \alpha)$. These satellites fall in the voltage range defined by $V_{1 n}$ and $V_{2 n}$ determined by

$$
V_{1 n} \pm n h \nu / m e=V_{10}
$$

and

$$
V_{2 n} \pm n h \nu / m e=V_{20} \text {. }
$$

It proved possible, however, to use the analysis leading to Eq. (8) only in a few cases, for instance, at the $m=1$ structures of Fig. 5. As is evident from the $m=1$ structures of Fig. 4 and the $m=2$ structures of Fig. 5, neighboring structures overlap making it meaningless to define $\Delta \sigma_{r f}$ as above. The approach via definition (5) proved adequate in such cases.

The background level is a sum of contributions, $\sigma_{m B}$, from the current carrying channels which are responsible for the SGS, i.e.,

$$
\sigma_{B}=\sum_{m} \sum_{n} J_{n}^{2}(m \alpha) \sigma_{m B},
$$

where we have used the relation ${ }^{33}$

$$
\sum_{n} J_{n}^{2}(m \alpha)=1
$$

Inserting Eqs. (3) and (9) into Eq. (5) and normalizing to $\Delta \sigma_{m}=\sigma_{m}\left(V_{10}\right)-\sigma_{m B}$ yields

$$
\frac{\Delta \sigma_{\mathrm{rf}}}{\Delta \sigma_{m}}=\sum_{n} J_{n}^{2}(m \alpha) \frac{\sigma_{m}\left(V_{1 n}+n h \nu / m e\right)-\sigma_{m B}}{\Delta \sigma_{m}} .
$$

Again Eq. (11) shows that, if only the $n$th term contributes at $V_{1 n}=(2 \Delta \pm n h \nu) / m e$, then the peak amplitude at $V_{1 n}$ varies as $J_{n}^{2}(m \alpha)$.

\section{Analyzed data}

The point-contact data of Fig. 4 were analyzed according to Eq. (11). Considering, first, the MAT around the gap voltage, the background level used was the conductance plateau on the high-voltage side of the gap peak. Furthermore, only peak amplitudes on this side of the gap peak were measured. Then, as is evident from Fig. 3(a), overlap effects are minimized. ${ }^{34}$ The measured normalized peak amplitudes for $n=0,1,2,3$, and 4 are shown in Fig. 9(a), 9(b), 9(c), 9(d), and 9(e), respectively, and compared to the corresponding squared Bessel functions $J_{n}^{2}(\alpha)$. The power scale 


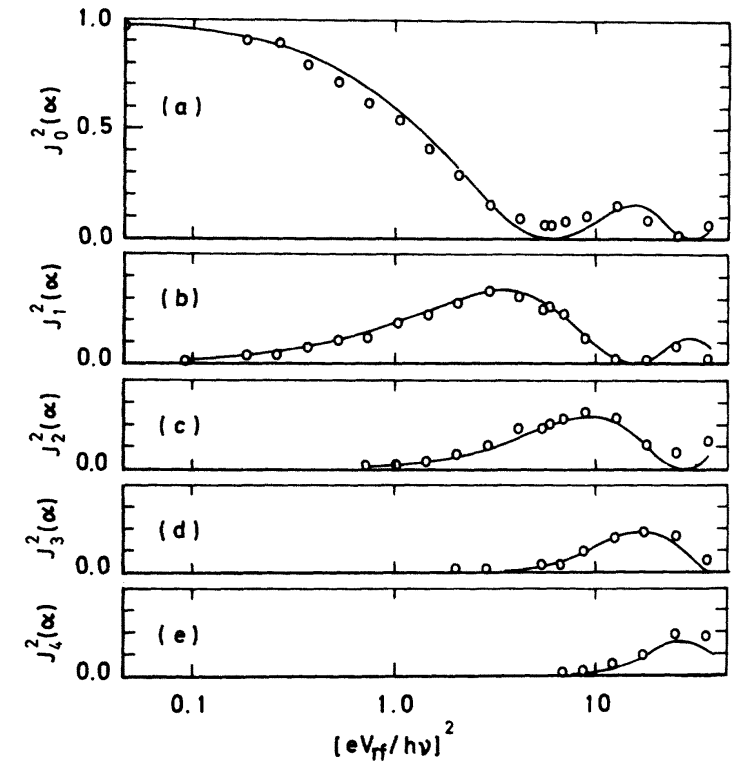

FIG. 9. Microwave-assisted tunneling results derived from the sequence of Fig. 4 (open circles) plotted as functions of the parameter $\alpha^{2}=\left(e V_{\mathrm{rq}} / h \nu\right)^{2}$, and compared with the squared Bessel functions shown as solid lines. The peak amplitudes are measured at voltages: (a) $2 \Delta / e$, (b) $2 \Delta / e+h v / e$, (c) $2 \Delta / e+2 h v / e$, (d) $2 \Delta / e+3 h v / e$, and (e) $2 \Delta / e+4 h \nu / e$.

is fixed for all the data by adjusting the scaling factor between the incoming $\mathrm{rf}$ power and the parameter $\alpha^{2}=\left(e V_{r f} / h \nu\right)^{2}$ so as to obtain the best fit of the experimental points in Fig. $9(b)$ to $J_{1}^{2}(\alpha)$ shown as the solid line. The agreement between theory and experiment is good establishing that the method of analysis is satisfactory.

The peak amplitudes of the $m=2$ and $m=3$ structures were also measured using as reference level the conductivity minimum at $V=1.6 \mathrm{mV}$ just above the $m=2$. The normalized results are shown in Fig. 10 together with the $m=1$ results of Fig. 9(a). Comparing the broken lines drawn through the experimental points with the squared zeroorder Bessel functions shown, of argument $2 \alpha$ and $3 \alpha$, respectively, the minima positions on the power axis are seen to coincide. A much better agreement could be obtained by adjusting the reference level. This was not done, however, because in order to separate out properly the contributions from the $n=0$ peaks from the satellites which are strongly overlapping, detailed calculations taking into account the shape of the peaks would be necessary. ${ }^{35}$

As already mentioned and as shown in Fig. 5 the resolution of satellites was improved by recording resistance rather than conductance. Figure 11 shows the analyzed results from two pointcontact junctions for which the resistance was re- corded. The open symbols and the $x$ 's derive from the data of Fig. 5 (junction 1) and the closed symbols and the +'s from another junction (junction 2). Before plotting the results, the measured amplitudes in resistance were converted to conductance. For the $m=1$ structures in both cases and for the $m=2$ structures of junction 2 the analysis was based on Eq. (8), and all the remaining structures were measured according to Eq. (11). For junction 1 the $m=2$ and $m=3$ structures were measured with the resistance plateau between the peaks as a reference [see also Fig. 3(b)], whereas for the $m=4$ peak the reference level was adjusted to produce fair agreement to theory showing nothing else but that consistent results could be obtained by a reasonable choice of reference.

Figure 11(a) shows the normalized amplitudes of the $n=0, m=1,2,3$, and 4 structures compared with the corresponding squared Bessel functions $J_{0}^{2}(m \alpha)$, and Fig. 11(b) shows the first satellites, i. e., $n=1$, for the $m=1$ and $m=2$ structures. The power scale is fixed by fitting to the points at the arrows in Fig. 11(a) for point-contact junctions 1 and 2 , respectively. Although the experimental points show deviations from the solid lines due to overlap effects which are not completely avoided, the results lead to the conclusion that the variation with $\mathrm{rf}$ power is consistent with the use of arguments $\alpha, 2 \alpha, 3 \alpha, \ldots$ in the Bessel functions.

The thin-film tunnel junction data of Fig. 7 and 8 have been analyzed and the measured normalized amplitudes are plotted in Fig. 12 as a function of rf power $\left(\alpha^{2}\right)$ for both the resistance and the conductance results (open and closed symbols, respectively). The resistance recordings were converted to conductance before amplitudes were measured and the analysis was then in both cases carried out as follows:

Conductance peaks were measured taking the background conductance level as a reference. That is, the $n=1$ peak was measured above the

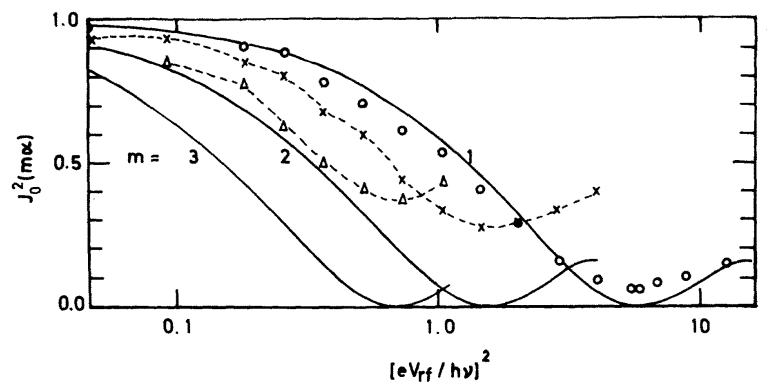

FIG. 10. Power dependence of the subharmonic gap structures of Fig. 4. $m=1$, circles. $m=2, \times$ 's. $m=3$, triangles. The solid lines are $J_{0}^{2}(m \alpha)$ for $m=1,2$, and 3 , respectively. 


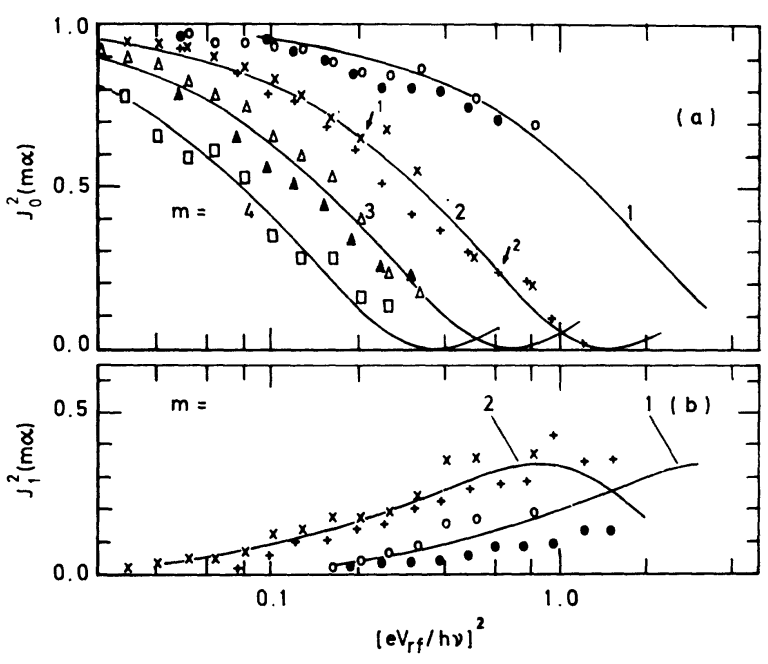

FIG. 11. Power dependence of the subharmonic gap structure of the rf-induced satellites derived from two $\mathrm{Nb}-\mathrm{Nb}$ point-contact junctions, open symbols and $x^{\prime}$ 's from the data of Fig. 5 (junction 1), closed symbols and +'s from another junction (junction 2). (a) Normalized amplitudes of the $n=0$ structures for $m=1$ (circles), $m=2$ ( $x$ 's and +'s), $m=3$ (triangles), and $m=4$ (squares). (b) Normalized amplitudes of the rf-induced satellites $(n=1)$ for $m=1$ and $m=2$. The symbols are as above. The solid lines show $J_{n}^{2}(m \alpha)$ with $n$ and $m$ as indicated. The power scale is fixed for all the curves by fitting to the points at the arrows marked 1 and 2 for junctions 1 and 2, respectively.

conductance level on the zero-rf-power trace at that particular voltage where the peak showed up, whereas the $n=0$ peak was measured from the conductance level-at the peak voltage-on the trace recorded at that particular power level where the peak amplitude reached its first zero. This is a well-defined level for the tunnel-junction measurements because here, the $n=0$ and the $n=1$ peaks do not overlap. Very detailed agreement has been obtained between the experimental results and the squared zeroth and first-order Bessel functions with arguments $\alpha$ and $2 \alpha$ shown in Fig. 12. Thus, this section can be concluded by stating that very strong experimental evidence has been presented that the $n$th structure belonging to the $m$ th-subharmonic series varies with rf power as $J_{n}^{2}(m \alpha)$ with $m=1,2,3, \ldots$ and $n=0,1,2, \ldots$.

The data presented in this section are our most detailed experimental results on the rf-power dependence. A number of other thin-film tunnel junctions (including a $\mathrm{Pb}-\mathrm{O}-\mathrm{Pb}$ junction) and pointcontact junctions were tested, however, less complete data were taken for a variety of reasons. It should be added, however, that all the junctions tested behaved in full agreement with the conclusion stated above.
D. Discussion of the microwave results

The materials ( $\mathrm{Nb}$ and $\mathrm{Sn}$ ) and the microwave frequency $(66 \mathrm{GHz})$ have been chosen with the purpose of having good resolution of the SGS and the rf-induced satellites. The resolution depends (i) on the spacing between the different $m$ structures, but also (ii) on their widths compared to the spacing, $h \nu / m e$, of the satellites. Niobium, having a large energy gap $(2 \Delta \simeq 3 \mathrm{mV})$, is for a fixed frequency favorable with respect to (i) but inferior compared to tin with respect to (ii). Therefore, by making measurements on $\mathrm{Nb}-\mathrm{Nb}$ point contacts as well as on $\mathrm{Sn}-\mathrm{O}$-Sn tunnel junctions, the $\mathrm{rf}$ dependence has been obtained (a) for high- $m$ structures with no overlap between satellites belonging to different $m$ series $(\mathrm{Nb})$, and (b) very accurately for the $n=0,1$ structures for low $m$ values $(\mathrm{Sn})$. The $\mathrm{rf}$ dependence thus established is the same for point-contact junctions as for thinfilm tunnel junctions. Only contact between mates of identical materials have been studied in the present work in order not to complicate the data analysis and the interpretation of the $\mathrm{rf}$ results with the problems of identifying the different structures.

The results presented in this section have been obtained on the most clean junctions among those tested. Occasionally additional structure, in the form of very sharp spikes in $d V / d I$, was observed in our point-contact junctions overlapping with the high- $m$ SGS's. ${ }^{36}$ The position of these structures

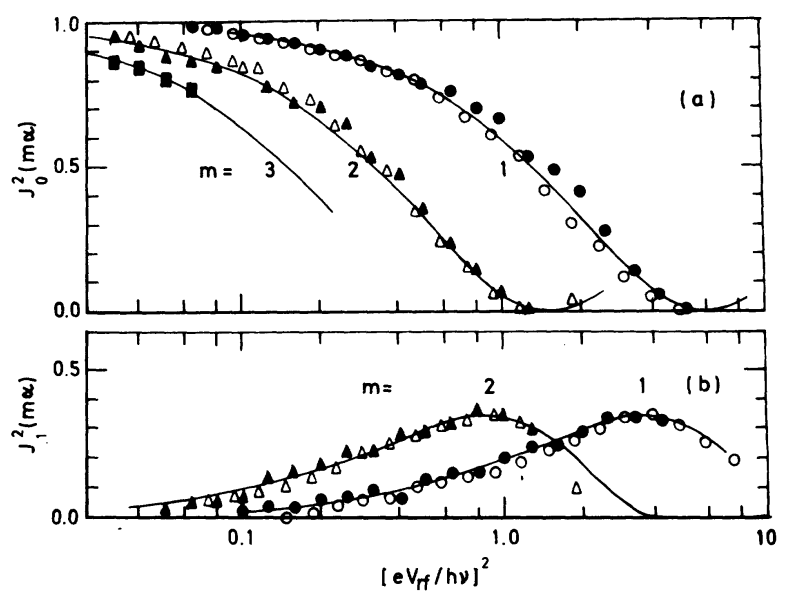

FIG. 12. Power dependence of the subharmonic gap structure and of the rf-induced satellites derived from two Sn-O-Sn tunnel junctions, open symbols from the data presented in part in Fig. 7, and closed symbols from the data presented in part in Fig. 8. (a) Normalized amplitudes of the $n=0$ structures for $m=1$ (circles), $m=2$ (triangles), and $m=3$ (squares). (b) Normalized amplitudes of the $\mathrm{rf}$-induced satellites $(n=1)$ for $m=1$ and $m=2$. The symbols are as above. The solid lines show $J_{n}^{2}(m \alpha)$ with $n$ and $m$ as indicated. 
on the voltage axis and their amplitude were dependent on the contact pressure. Furthermore, as rf was applied side peaks showed up at distances $n h v / 2 e$ from the center peak, independent of its position. These observations lead to the conclusion that their origin was a quarterwave resonator formed by a bent point. ${ }^{37}$ Complete rf measurements were, however, only carried through if the SGS appeared free of such "parasitic" structures. We are, therefore, confident that unambiguous data have been obtained showing that the SGS and the satellites vary with rf power as described by the squared Bessel functions $J_{n}^{2}(m \alpha)$.

Previous publications have discussed whether the appearance of two essentially distinct shapes of the SGS should be interpreted as evidence for two or more processes being responsible for the $\mathrm{SGS}^{5,8,11,12,14}$ or if the self-coupling mechanisms of Werthamer, ${ }^{18}$ in spite of this apparent distinction, explain all the structure. ${ }^{11}$ We have observed both shapes in question, i.e., peaks and steps in the current, and will return to that in Sec. IV.

Here, we want only to stress the fact that independent of the actual line shape of the SGS at a particular voltage $2 \Delta /$ me, this shape was replicated by the $r f$-induced satellites spaced by $\mathrm{hv} / \mathrm{me}$, and their power dependence was invariably found to be as $J_{n}^{2}(m \alpha)$.

In the following paper, Hasselberg et al. ${ }^{22}$ consider multiparticle tunneling and self-coupling mechanisms as the origin of the SGS. In all cases considered the rf-power dependence of the structures-both SGS and satellites-are found to agree with the experimental results presented here.

\section{DISCUSSION OF OTHER RESULTS}

The microwave dependence of the SGS established by this work does not distinguish multiparticle tunneling from nonlinear self-coupling. Others ${ }^{8,11,12,14}$ have, following the suggestion of Werthamer, ${ }^{18}$ attempted to establish such a distinction between the theories by using contacts between dissimilar superconductors. With the exception of Giaever and Zeller, ${ }^{11}$ who interpreted their results as support for self-coupling mechanisms, these authors conclude that no satisfactory theory has been published or that no choice could be made between the existing theories on the basis of the experiments.

In this section we review and discuss aspects of our experimental results other than the rf dependence. Since a distinction between the theories may be based on properties such as line shapes and relative amplitudes of the SGS, ${ }^{22}$ these features will be studied more closely.

Particularly for the point-contact junctions, where the $I V$ characteristic is easily adjusted, the SGS may take a variety of forms. Figure 2 shows some typical examples. As also noted by others $\mathrm{s}^{5,8,11,12,14}$ two characteristic and distinctly different shapes are observed, namely, current cusps (peaks) and current steps. It was first suggested by Marcus ${ }^{5}$ that current cusps and current steps might be evidence for self-coupling mechanisms and multiparticle tunneling being dominant, respectively. This need not be the case according to the following observations from Fig. 2:

(a) As the contact pressure was increased the contact resistance decreased and the zero-voltage current increased. At the same time the shape of the SGS changed gradually from steps to cusps starting with the high $-m$ structure. In Fig. 2(a) all the structures are current steps. In Fig. 2(b) the $m=1$ structure is a current step, whereas the higher $-m$ structures have become more cusplike. Finally, in Fig. 2(c), the change to current cusps is complete.

(b) By a closer inspection of Fig. 2(a) we find, first, that the $m=2$ structure is very large compared to previously reported "two-particle tunneling processes," $1-3,8$ the step amplitude being about one-third of the single-particle tunneling step ( $m$ $=1$ ); second, that the $m=3$ structure is missing on the current scale used; and third, that the $m=4$ structure can be seen as a very tiny current step on the same current scale.

The analysis of Hasselberg et al..$^{22}$ predicts, in the case of self-coupling mechanisms, that for the odd series ( $m=$ odd integer) the line shape can be a step, a peak, or any superposition of such shapes, depending on the coupling strength. Furthermore, they show that weak self-coupling favors steps, that the coupling strength increases with increasing zero-voltage current and also with decreasing bias voltage, and finally, that self-coupling predicts amplitudes of the SGS which decreases much more slowly with increasing $m$ than does multiparticle tunneling. Observations (a) are qualitatively consistent with these analyses. The theoretical analysis of the even- $m$ series ( $m$-even integer) is not equally detailed. Within the self-coupling formulation this series should be due to pairbreaking in the superconductors caused by the internal electromagnetic fields generated by the ac Josephson current. As discussed by Hasselberg et al., ${ }^{22}$ the even- $m$ series due to pair breaking can-with respect to line shapes and relative amplitudes-very well be similar to the odd- $m$ series although their origins are conceptually different. We do not know at present whether the apparently smooth decrease of the SGS amplitudes with increasing $m$, i.e., combining the odd and even series into one, is inherent in the self-coupling formulation.

The observations (b) that the $m=3$ structure is 
missing in Fig. 2(a) and that the $m=2$ and 4 structures are seen certainly disagrees with multiparticle tunneling as being the sole responsible process. Pure multiparticle tunneling should produce structures which decrease monotonically with increasing $m$ at a much faster rate than observed in any of our junctions. In fact, the presence of the $m=2$ and 4 structures unaccompanied by the $m=3$ and 5 structures may be significant as an indication that the mechanisms producing the even and odd series are different. ${ }^{38}$ Hence, none of our experimental results are in contradiction to the self-coupling theory, whereas multiparticle tunneling alone can definitely not explain the SGS.

Within the self-coupling formulation supplementary processes to the odd- $m$ tunneling processes have been suggested. ${ }^{22}$ If very close coupling exists between the two superconductors of the junction, then pair-breaking processes become possible where one quasiparticle is created in each superconductor. Thus, pair breaking alone may produce structure for all $m$, structure which does not depend on the observation of the quasiparticle-tunneling threshold at $2 \Delta / e$. Very close coupling implies extremely thin tunneling barrier or else metallic contact in the form of bridges connecting the superconductors. Such bridges were certainly present in the point-contact junction of Fig. 2(c) $)^{39}$ and the SGS, including the $m=1$ structure, might be dominated in this case by such pair-breaking processes. Metallic bridges may not be required, however, in order to see SGS. For example, the tunnel junction of Fig. 1 was - to the best of our knowledgefree from such bridges. First, because the zerovoltage current could be suppressed completely by a small magnetic field (leaving the SGS unchanged), and second, because the magnitude of the zerovoltage current was equal to 0.9 times the current step at $2 \Delta / e$ as predicted by theory. ${ }^{40}$

The often quite complicated SGS line shapes can, as discussed above, result from superposition of peak and step structure elements. This implies that a proper indexing of the structure can not be made without a more detailed study of the selfcoupling problem. Various authors have used different indexing criteria. For example, indexing has been attempted using resistance dips (conductance maxima) ${ }^{11}$ as used conventionally for the single-particle tunneling step at the gap voltage following the suggestion of Townsend and Sutton. ${ }^{41} \mathrm{Re}-$ sistance peaks have also been chosen to index the structures, ${ }^{8,12}$ and even inflection points on the high-voltage side of conductance peaks (or equivalently on the low-voltage side of resistance peaks) have been suggested to be most reasonable if the structures are current peaks. ${ }^{9,10,12,14}$ We have tested all these methods on our junctions and have found as a general trend but with considerable scat- ter that independent of the indexing criteria used, the energy gap $2 \Delta / e$ determined by $m V_{m}$, where $V_{m}$ is the indexing voltage, decreases as $m$ increases. This agrees with the findings of others. ${ }^{8-10,12,21}$

The high-resistance regions on the high-voltage side of the current cusps seen when constant-current bias was used reflect the loadline of the bias circuitry rather than the true line shape of the SGS. This is clearly displayed in Fig. 6. The high-resistance regions appear in the derivative $d V / d I$ as narrow peaks, and the resolution obtained for the satellite structures of our $\mathrm{Nb}-\mathrm{Nb}$ point contacts result from this artificial sharpening of the structure when measured in resistance. It is evident also that this effect further complicates the indexing since the true line shapes can not be traced out.

\section{SUMMARY AND CONCLUSION}

The microwave dependence of the so-called subharmonic gap structure has been studied experimentally. Based on the qualitative behavior and by analogy to the well-known microwave-assisted tunneling effect of small junctions, an analytical expression describing the microwave response has been postulated. From this expression a data-reduction procedure has been derived which was tested on microwave-assisted tunneling and then used on the subharmonic gap structure. The results thus obtained can be summarized as follows:

As microwaves are applied the amplitude of the subharmonic gap structure at the voltage $2 \Delta / m e$ varies with microwave power as the squared zeroorder Bessel function $J_{0}^{2}(m \alpha)$, of argument $m \boldsymbol{x}$, where $\alpha$ is related to the microwave voltage drop $V_{\mathrm{rf}}$ across the junction by $\alpha=e V_{\mathrm{rf}} / h \nu$. Furthermore, replicas of the $m$ th subharmonic gap structure grow up on both sides-at voltages $(2 \Delta \pm n h \nu) / m e$, where $n$ $=1,2,3, \ldots-$ with amplitudes varying as squared Bessel functions, $J_{n}^{2}(m \alpha)$, of orders $n$ and of argument $m \alpha$.

In parallel to the experimental work presented here Hasselberg, Levinsen, and Samuelsen ${ }^{22}$ made a theoretical analysis of various explanations of the subharmonic gap structure. The result of their analysis is that both explanations considered, multiparticle tunneling and self-coupling, yield the experimentally observed power dependence. Hence, a distinction between the theories must rely on other, less obvious properties such as line shapes and relative amplitudes. We have reviewed ou $r$ data accordingly and have found strong evidence that multiparticle tunneling must be discarded as the sole explanation of the structure, whereas selfcoupling mechanisms seem able to account for all our observations.

\section{ACKNOWLEDGMENT}

We gladly acknowledge the interest and encouragement of Professor K. Saermark at all stages of this work. 
*Permanent address: Department of Electrical Engineering, University of Rochester, N.Y. 14627.

${ }^{1}$ B. N. Taylor and E. Burstein, Phys. Rev. Lett. 10, 14 (1963).

${ }^{2}$ C. J. Adkins, Philos. Mag. $\underline{8}, 1051$ (1963).

${ }^{3}$ C. J. Adkins, Rev. Mod. Phys. 36, 211 (1964).

${ }^{4}$ I. K. Yanson, V. M. Svistunov, and I. M. Dmitrenko, Zh. Eksp. Teor. Fiz. 47, 2091 (1964) [Sov. Phys. JETP 20, 1404 (1965)].

${ }^{5}$ S. M. Marcus, Phys. Lett. 19, 623 (1966).

${ }^{6}$ S. M. Marcus, Phys. Lett. 20,236 (1966).

${ }^{7}$ G. I. Rochlin, Phys. Rev. $1 \frac{153}{}, 513$ (1967).

${ }^{8}$ J. M. Rowell and W. L. Feldman, Phys. Rev. 172,393 (1968).

${ }^{9}$ I. K. Yanson and Kh. Albegova, Zh. Eksp. Teor. Fiz. 55, 1578 (1968) [Sov. Phys. -JETP 28, 826 (1969)].

${ }^{10}$ A. A. Bright and J. R. Merrill, Phys. Rev. 184, 446 (1969).

${ }^{11}$ I. Giaever and H. R. Zeller, Phys. Rev. B $\underline{1}, 4278$ (1970).

${ }^{12}$ S. Bermon and R. M. Mesak, Solid State Commun. $\underline{9}$, 2143 (1971).

${ }^{13}$ H. J. Levinstein and J. E. Kunzler, Phys. Lett. 20, 581 (1966).

${ }^{14}$ L. J. Barnes, Phys. Rev. 184, 434 (1969).

${ }^{15}$ A. Longacre, Jr. and S. Shapiro, Bull. Am. Phys. Soc. 16, 399 (1971).

${ }^{16}$ M. Puma and B. S. Deaver, Jr., Appl. Phys. Lett. 19,539 (1971).

${ }^{17}$ J. R. Schreiffer and J. W. Wilkins, Phys. Rev. Lett. 10,17 (1963).

${ }^{18}$ N. R. Werthamer, Phys. Rev. 147, 255 (1966).

${ }^{19}$ A. Zawadowski, Phys. Lett. 23, 225 (1966).

${ }^{20}$ S. Strässler and H. R. Zeller, Phys. Rev. B $\underline{3}, 226$ (1971).

${ }^{21}$ Very recently Gregers-Hansen et al., have reported observation of SGS and rf-induced satellites (sidebands) in superconducting microbridges near $T_{c}$. The $\mathrm{rf}$ dependence of these structures is consistent with our results on point contracts and tunnel junctions in that the sensitivity to $\mathrm{rf}$ increases with the number $m$. A quantitative comparison with squared Bessel functions has, however, not been made. [P. E. Gregers-Hansen, E. Hendriks, M. T. Levinsen, and G. R. Pickett, Phys. Rev. Lett. 31, 524 (1973)].

${ }^{22}$ L. -E. Hasselberg, M. T. Levinsen, and M. R. Samuelsen, following paper, Phys. Rev. B 9,3757 (1974).

${ }^{23}$ B. Kofoed, B. U. Jensen, and K. Saermark (unpublished).

${ }^{24} \mathrm{~J}$. W. Dozier and J. D. Rodgers, IEEE Trans. Microwave Theory Tech. 12,360 (1964); IEEE Trans. Microwave Theory Tech. $\overline{12}, 572$ (1964).

${ }^{25} \mathrm{C}$. A. Hamilton and $\mathrm{S}$. Shapiro, Phys. Rev. B $\underline{2}, 4494$ (1970).

${ }^{26}$ M. R. Samuelsen, B. Kofoed, and O. Hoffmann Soerensen, Phys. Status Solidi A 11, K79 (1972).

${ }^{27}$ O. Hoffmann Soerensen (unpublished).

${ }^{28}$ A. H. Dayem and R. J. Martin, Phys. Rev. Lett. 8 , 246 (1962).
${ }^{29}$ P. K. Tien and J. P. Gordon, Phys. Rev. $\underline{129}, 647$ (1963).

${ }^{30}$ O. Hoffmann Soerensen and M. R. Samuelsen, Phys. Lett. A 39, 137 (1972).

${ }^{31}$ Conditions under which Eq. (2) might be expected to apply are discussed from a theoretical point of view in the companion paper by Hasselberg, Levinsen, and Samuelsen (Ref. 22).

${ }^{32}$ Note that the characteristic structure element, although associated with the $2 \Delta /$ me structure does not necessarily fall at this voltage. Note also, that the normalization is only reasonable if we assume that, in the zero power limit, the measurable quantity $\Delta \sigma_{\mathrm{rq}}$ at the subharmonic in question equals $\Delta \sigma_{m}$.

${ }^{33} \mathrm{M}$. Abramowitz and I. A. Stegun, Handbook of Mathematical Functions, 5th ed. (Dover, New York, 1964). Formula No. 9.1.76.

${ }^{34}$ The line shape of the gap peak is such that the $n$th peak does not contribute at the voltage where the $(n+1)$ st peak is measured. However, the $(n+2)$ nd peak does contribute here, and this explains the systematic deviations between the experimental results and the Bessel functions at power levels where the $(n+2)$ nd peak has attained measurable amplitude.

${ }^{35}$ Measurements on thin-film tunnel junctions at 3 and 10 $\mathrm{GHz}$ have been carried out. Here $h \nu$ is small compared to the width of the structures and calculations taking the peak width into account in MAT will be reported (B. Kofoed, K. Saermark, and U. K. Poulsen, report in preparation).

${ }^{36}$ Microwave-induced Josephson steps were also seen, but the experiments were stopped when these steps entered into the voltage region of interest. This is not unreasonable in view of the condition $V_{\mathrm{rf}} \ll V_{\mathrm{dc}}$ in $\mathrm{Ap}-$ pendix II of Ref. 22.

${ }^{37} \mathrm{~A}$. Longacre, Jr. and Sidney Shapiro, in Proceedings of the Symposium on Submillimeter Waves, Microwave Research Symposia Series, edited by J. Fox (Polytechnic Press, Brooklyn, New York, 1971), Vol. XX, p. 295.

${ }^{38}$ That the zero-voltage current is missing in Fig. 2 (a) doe not imply that no ac fields are excited in the junction. This has been very elegantly demonstrated by $\mathrm{L}$. L. Vant-Hull and J. E. Mercereau [Phys. Rev. Lett. 17,629 (1966)].

${ }^{39}$ The zero-voltage current showed small amplitude oscillatory behavior as a function of magnetic field typical of weak links in parallel if their critical currents are different.

${ }^{40}$ A. I. Larkin and Yu. N. Ovchinnikov, Zh. Eksp. Teor. Fiz. 51, 1335 (1966)[Sov. Phys. -JETP 24, 1035 (1967)]. Here it is shown that, for identical superconductors, both the maximum zero-voltage current and the magnitude of the current step at $2 \Delta / e$ equals $\left(\pi \Delta / 2 e R_{N}\right)$ $\tanh (\Delta / 2 k T)$. The factor 0.911 enters if strong-coupling effects are included, see, e.g., T. A. Fulton and D. E. McCumber [Phys. Rev. 175, 585 (1968)].

${ }^{41} \mathrm{P}$. Townsend and J. Sutton, Phys. Rev. 128, 591 (1962). 